\title{
Editorial
}

Pathobiology

Pathobiology 2009;76:53-54

DOI: $10.1159 / 000201673$

\section{Molecular Basis of Disease Prevention}

It is well accepted that numerous diseases can be prevented by life style interventions. This holds especially true for several types of cancers such as colorectal cancer, skin cancer or lung cancer. The recent WHO Health Report claims that up to $40 \%$ of the worldwide cancer burden could be prevented by adopting healthier life styles. The epidemiological evidence for this has been established for a long time. Molecular epidemiology tried to link epidemiology and molecular markers that are specific for given cancers.

Cohort studies and intervention studies have managed to show that for instance the intake of fruits and vegetables can reduce the incidence of colon cancer. There are now increasing possibilities to explain the molecular basis of these epidemiologically proven facts. Molecules that interact with basic cellular functions such as apoptosis, proliferation and differentiation are the most interesting ones.

The studies in this issue of Pathobiology try to describe the state of the art knowledge of the molecules behind cancer prevention. There are possibilities of using these substances as biomarkers, either prognostic or diagnostic; in addition, further understanding will enable us to better use naturally occurring products and/or to avoid substances in diet or therapy.

The influence of diet has been demonstrated for several cancers. Generally speaking, a diet rich in fruits, vegetables, fish and low in red meat and saturated fats is considered to lower the risk of developing colorectal cancer and to prevent several other diseases. Until now, these data were mainly collected by epidemiologists, but the biological substrate for successful prevention has remained unexplained. Ding et al. in this issue review how phytochemicals exert their beneficial effects at the cellular level. Among these dietary molecules, chemicals such as terpenoids, polyphenolics, and organosulfurous and alkaloid compounds have received considerable attention. They frequently act as insect repellents for plants, whereas in humans, they increase the level of cellular reaction to stress/inflammation. This mechanism is called hormesis and may operate in several other situations. To achieve such results phytochemicals must interfere with the cell signaling mechanisms. This can happen at the cell membrane itself, further downstream in the pathways or, finally, at the cellular nucleus. The authors of this review clearly show that the many pathways that are modified by phytochemicals slow down the inappropriately proliferating cells.

In cervical cancer the situation is quite different: over $90 \%$ of all cervical carcinomas are linked to oncogenic subtypes of the human papillomavirus. For this virus a model of interaction with the epithelial cell has been established: an important step of viral transformation is the overexpression of the viral oncogenes in basal epithelial cells. This leads to uncontrolled activation of the cell cycle, to restriction of apoptosis, and finally to chromosomal instability with viral integration. HPV is an important part of the oncogenic process and the several molecules identified that take part in this transformation may well be used as molecular markers of the neoplastic process. Still, the following questions remain: Could these molecular markers replace the current cervical cancer

\begin{tabular}{ll}
\hline KARGER & (c) 2009 S. Karger AG, Basel \\
1015-2008/09/0762-0053\$26.00/0 \\
$\begin{array}{l}\text { Fax +41 61 306 } 1234 \\
\begin{array}{l}\text { E-Mail karger@karger.ch } \\
\text { www.karger.com }\end{array}\end{array}$ & $\begin{array}{l}\text { Accessible online at: } \\
\text { www.karger.com/pat }\end{array}$
\end{tabular}


screening? Will there be other molecular markers or combinations of them that will finally render a molecular prediction of cervical cancer possible? Wentzensen and Klug discuss this topic.

Skin cancer and particularly melanoma is one of the key cancers for which primary prevention consists in avoiding UV radiation. Almost all skin cancers which were detected early are curable. There are biological and epidemiological differences among malignant melanoma, basal cell carcinoma and squamous cell carcinoma. The review of Greinert et al. considers all these situations and discusses part of the information that has been collected in the fields of genetics, molecular pathways and that relates to cellular changes which are important indicators in skin cancer induction, promotion, progression and metastasis. The data derived from gene expression profiling shows that there are biomarkers for use as exposure markers, markers of external dose and markers of biologically effective dose, markers of altered structure/ function, markers of susceptibility and epigenetic markers. All this newly accumulated knowledge can be of po- tential use for facilitating primary prevention, allowing identifying persons at risk and early stages of skin cancer.

Breast cancer is in a large number of cases a hormonedependent neoplasia. The role and function of naturally occurring as well as artificially produced hormones have been studied. Is the use of hormones or hormone-like substances a way of changing the incidence of breast cancer? There are already studies underway looking at the 'protective' effect of antihormones to be taken preventively by women with a family risk of breast cancer. On the other hand, there are millions of women worldwide taking hormones to alleviate menopausal symptoms such as hot flashes or dryness of the mucosae. Since the publication of the Women Health Initiative, changes in hormone therapy have occurred. This is a large epidemiological 'cohort' that could also answer questions as to the link between breast cancer prevention and hormones. The group of Katalinic reports on their recent experience in Germany.

Bettina Borisch, Geneva 